\title{
An info-gap application to robust design of a prestressed space structure under epistemic uncertainties
}

\author{
Aurélien Hot ${ }^{\mathrm{a}}$, Thomas Weisser ${ }^{\mathrm{b}, *}$, Scott $\operatorname{Cogan}^{\mathrm{c}}$ \\ ${ }^{a}$ Centre National d'Études Spatiales, 18 Avenue Édouard Belin, 31401 Toulouse Cedex 9, France \\ ${ }^{\mathrm{b}}$ Laboratoire MIPS, Université de Haute-Alsace, 12 rue des frères Lumière, 68093 Mulhouse, France \\ ${ }^{\mathrm{c}}$ Département Mécanique Appliquée, Institut FEMTO-ST, 24 chemin de l'Épitaphe, 25000 Besançon, France
}

\section{A R T I C L E I N F O}

\section{Article history:}

Received 8 October 2015

Received in revised form 24 September

2016

Accepted 16 December 2016

\section{Keywords:}

Epistemic uncertainty

Info-gap theory

Robustness

Prestressed structure

Mechanical contact

\begin{abstract}
A B S T R A C T
Uncertainty quantification is an integral part of the model validation process and is important to take into account during the design of mechanical systems. Sources of uncertainty are diverse but generally fall into two categories: aleatory due to random process and epistemic resulting from a lack of knowledge. This work focuses on the behavior of solar arrays in their stowed configuration. To avoid impacts during launch, snubbers are used to prestress the panels. Since the mechanical properties of the snubbers and the associated preload configurations are difficult to characterize precisely, an info-gap approach is proposed to investigate the influence of such uncertainties on design configurations obtained for different values of safety factors. This eventually allows to revise the typical values of these factors and to reevaluate them with respect to a targeted robustness level. The proposed methodology is illustrated using a simplified finite element model of a solar array.

(c) 2016 Elsevier Ltd. All rights reserved.
\end{abstract}

\section{Introduction}

In the field of structural dynamics, the mathematical model depicting a real mechanical system must not only be validated against available test data, but also with respect to its final performance in the presence of uncertainties. Indeed, the required performance may be drastically affected even by a small perturbation of the design hypotheses such as the nominal model properties. Moreover, real environmental conditions and loads acting on a structure are usually unknown and are generally not accurately reproduced during tests. It is thus necessary to take these uncertainties into account in the design process.

This work focuses on the robust design of prestressed space structures such as solar arrays encountered on satellites (Fig. 1). During the spacecraft launch phase these are stowed in their folded configuration to save space under the launch vehicle fairing to reduce the risk of damage. Solithane snubbers (i.e. shock absorbers) are inserted between two adjacent panels to introduce a prestress and to absorb vibrations. However, under high excitation loads, a loss of contact may occur resulting in impacts which may damage both solar generators and fragile on-board equipment [1]. In practice, the specific load configuration for which the separation of two neighboring panels occurs is difficult to determine precisely since the exact level of prestress applied to the structure is uncertain.

Several classifications for the sources and types of uncertainties encountered in mechanical design problems can be found in the literature (e.g. in [2,3]). It is common in structural dynamics to distinguish two classes of uncertainties. The first one,

\footnotetext{
* Corresponding author.

E-mail address: thomas.weisser@uha.fr (T. Weisser).
} 


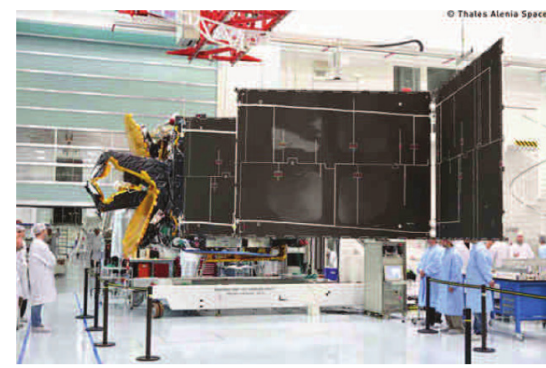

(a) Opening test

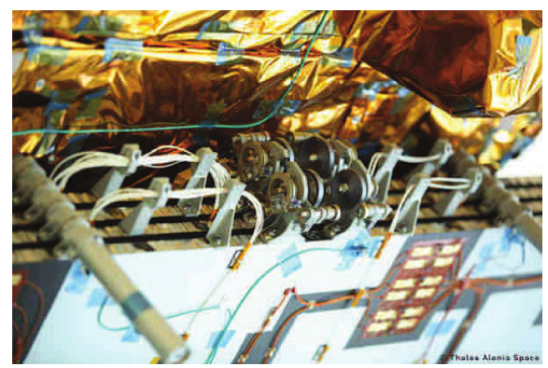

(b) Complex connexions between panels

Fig. 1. Views of solar generators on satellites.

called aleatory uncertainties, is due to the intrinsic randomness of the structure parameters such as the variability of its physical properties (e.g. Young's moduli, densities,...), of the manufacturing and assembling processes, of its service conditions (e.g. temperature, hydrometry,...), but also the variability of the experimental measuring process. The second class pertains to epistemic uncertainties. These result from a lack of knowledge or erroneous assumptions such as unknown values of junction equivalent stiffnesses, simplifying linearity hypothesis, omission of nonlinear or contact elements, unknown probability distributions of properties,.. .

It is common practice to use probabilistic methodologies [4] to quantify and to propagate aleatory uncertainties through the system model. A classical approach consists in using a deterministic finite element model and further adding uncertainties through either parametric or non-parametric methods. In parametric methods, the uncertain physical parameters are characterized by their mean value, their standard deviation or by probability density functions (e.g. Gaussian or uniform distributions). In non-parametric methods, model uncertainties are handled by directly introducing the uncertainties via global randomized matrices through dispersion parameters [5].

However, probabilistic approaches are not necessarily appropriate to treat all kinds of uncertainty. This is particularly true for epistemic uncertainties where the important information is simply missing. Non-probabilistic methods have thus been proposed including, for example, interval analysis [6,7], fuzzy sets theory [8,9] or lack-of-knowledge theory [10]. Ben-Haim initially proposed in [11] an approach dedicated to problems subjected to sever uncertainties, referred to as the info-gap theory: its basic concept consists in investigating the degree of lack of knowledge that can be tolerated while still satisfying a given critical level of performance.

This paper proposes to investigate the case of epistemic uncertainties due to lack of knowledge in the prestress level using an info-gap approach, in order to determine a robust design for solar arrays in their stowed configuration. The remainder of this article is organized as follows. In Section 2, a simplified finite element model of a solar array system is presented and its dynamic behavior is described. In Section 3, the general framework of an info-gap analysis is given and each of the associated components is further detailed within the scope of the previously described design problem. Finally, based on the derived indicators, the robustness of different design configurations is compared and the impact of the applied safety factor is investigated.

\section{Simplified model of solar array system}

As mentioned in the introduction, during the launch phase, spacecraft are subjected to a harsh dynamic environment. When large solar arrays are in their stowed configuration, impacts are thus likely to occur between two adjacent panels. In order to limit such phenomena and to avoid any resulting damage, dedicated snubbers are usually inserted between the panels, leading to the following modifications: firstly, an increase of the local stiffness, thus limiting the relative displacements; secondly, an induced prestress force, ensuring that contact is maintained between both panels. However, for high input levels, loss of contact between the snubbers and the panels may arise and impacts may still occur.

\subsection{Finite element model}

A finite element model of a simplified solar array has been developed in MSC-NASTRAN to investigate this problem, as depicted in Fig. 2. It consists in two plates (perpendicular to the $z$ direction), meshed with 3072 elements (CQUAD4). The three stacking points are modeled using beam elements (CBEAM) that are linked to the plates through rigid body elements (RBE2). While neglecting the mass of these junctions, the masses associated with both upper hinges are taken into account using concentrated mass elements (CONM2), also linked to the plates by rigid body elements.

The associated element properties are given in Table 1, where $E, \rho, v, T, d$ and $m$ represent the Young's modulus, the Poisson's ratio coefficient, the plate thickness, the beam cross section and the concentrated mass values, respectively. Finally, the snubbers' dynamic behavior is modeled by two linear springs inserted between points 1-2 and 3-4, acting along the global $z$-direction and whose stiffness values are initially equal to $k_{s}=1 \times 10^{5} \mathrm{~N} \mathrm{~m}^{-1}$. 


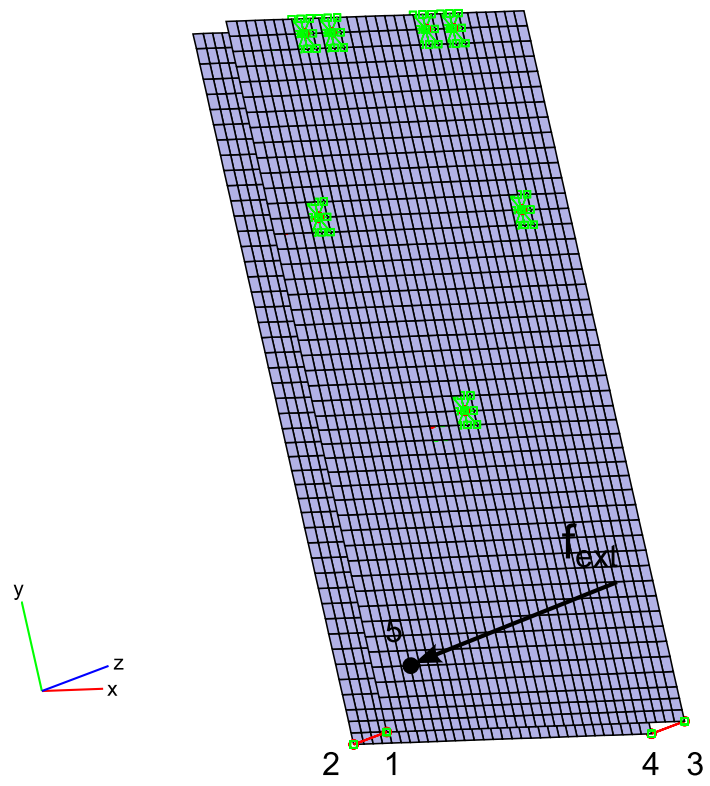

Fig. 2. Finite element model of a simplified solar array.

Table 1

Element properties of the model.

\begin{tabular}{llll} 
CQUAD4 & $E=6.52 \times 10^{10} \mathrm{~Pa}$ & $\rho=2.761 \mathrm{~kg} \mathrm{~m}^{-3}$ & $T=0.34$ \\
CBAR & $E=5.18 \times 10^{10} \mathrm{~Pa}$ & $\rho=3.453 \mathrm{~kg} \mathrm{~m}^{-3}$ & $v=0.34$ \\
CONM2 & $m=191 \times 10^{-3} \mathrm{~kg}$ & & $d=16 \times 10^{-3} \mathrm{~m}$ \\
\hline
\end{tabular}

\subsection{Dynamic behavior}

A sine excitation $f_{\text {ext }}$ of unitary amplitude is initially applied at node 5 (Fig. 2) in the $z$-direction. The corresponding acceleration response function ${ }^{1}$ at node 1 along the $z$-direction is plotted in Fig. 3(a). Resonances can be observed, especially at $62 \mathrm{~Hz}, 64 \mathrm{~Hz}, 89 \mathrm{~Hz}$ and $111 \mathrm{~Hz}$ corresponding the four first normal vibration modes, respectively. The associated deflection fields are displayed in Fig. 4.

As seen in Fig. 4(a) and (b), the first two deflection fields correspond to in-phase modes of the plates. The following mode shapes have out-of-phase relative displacements between opposite corners, implying a local strain of the interface stiffnesses. The generated local reaction force $f_{\text {reac }}$ at node 1 can be determined using the MPCF NASTRAN card, as displayed in Fig. 3(b).

\section{Info-gap analysis}

\subsection{General overview}

The info-gap theory proposed by Ben-Haim aims to quantify the robustness of model-based decisions to lack of knowledge in the system model or the performance requirement from the system [12]. This approach provides a useful framework to investigate the trade-off between performance and robustness but also to compare different candidate design solutions. Considering the general formulation detailed below, it has been used in a variety of fields, and in particular in structural dynamics concerning, for example, model updating [13,14], reliability analysis [15,16], optimal test design [17], robust design in civil engineering regarding seismic activity [18].

An info-gap analysis aims to quantify the difference (i.e. the gap) between the information that is known, e.g. the nominal value of a parameter, and the information that has to be known in order to ensure an a priori defined critical level of performance. An info-gap analysis is based on the definition of four components:

\footnotetext{
${ }^{1}$ The whole structure has been a priori submitted to a proportional damping assumption, i.e. $\boldsymbol{C}=\alpha \boldsymbol{K}+\beta \boldsymbol{M}$, where $\boldsymbol{M}, \boldsymbol{K}$ and $\boldsymbol{C}$ correspond to the global assembled mass, stiffness and damping matrices of the structure, respectively, and $\alpha=1 \times 10^{-5}$ and $\beta=2$.
} 


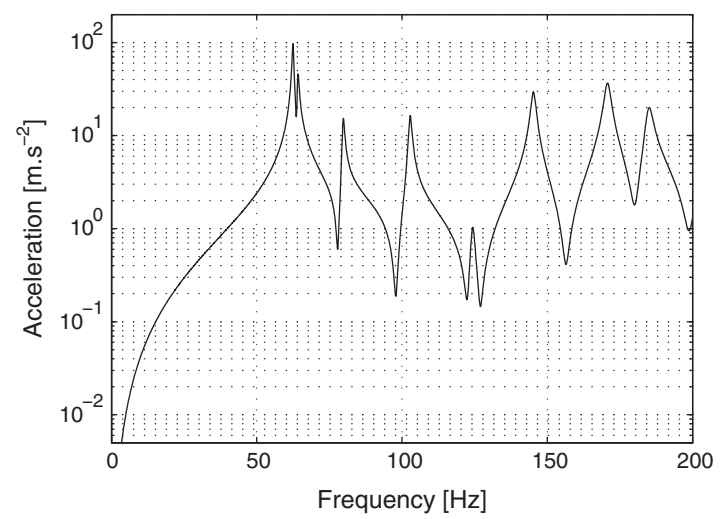

(a) Acceleration response

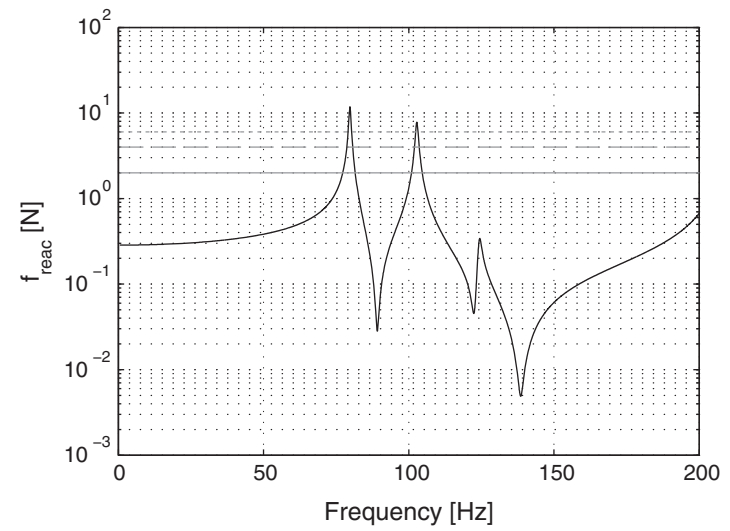

(b) Reaction force $f_{\text {reac }}$;

prestress levels $f_{p s}=(-) 2 N,(=-) 4 N,(\cdots) 6 N$

Fig. 3. Dynamic behavior of the structure at node 1 .

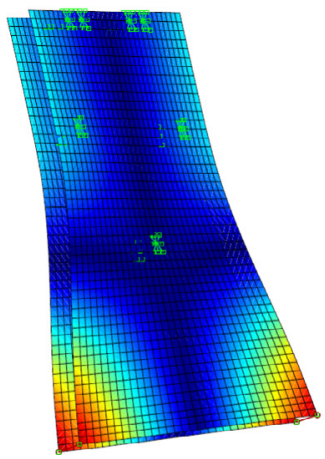

(a) $62 \mathrm{~Hz}$

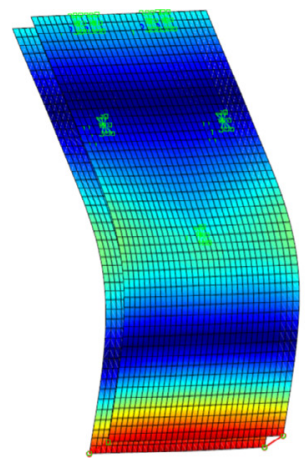

(b) $64 \mathrm{~Hz}$

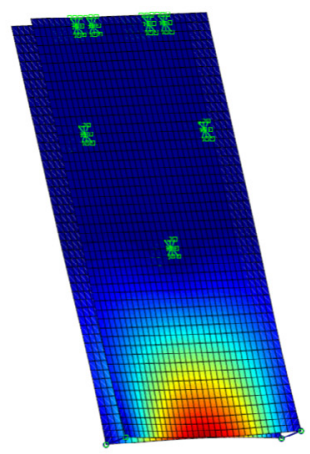

(c) $89 \mathrm{~Hz}$

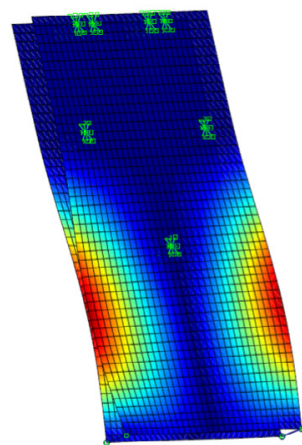

(d) $111 \mathrm{~Hz}$

Fig. 4. Deflection fields of the first normal modes.

- The system model, denoted $\mathcal{S}(\boldsymbol{q})$, depending on various unknown parameters $\boldsymbol{q}$, and defining the relation between the system inputs and outputs. In the case of structural dynamics, it usually comes down to the equation of motion, linking the external loads to the system responses (displacements, velocities, or accelerations) via the finite element model.

- The uncertainty model, denoted $\mathcal{U}\left(\boldsymbol{q}_{\boldsymbol{o}}, h\right)$, representing the uncertainty associated with the variable $\boldsymbol{q}$. It consists in a collection of nested sets of uncertain events centered on $\boldsymbol{q}_{\boldsymbol{o}}$ whose size is controlled by a parameter $h$, also referred to as the horizon of uncertainty: the larger this horizon, the more inclusive the uncertainty model. 
- The performance requirement, corresponding to the specification of a critical level, denoted $P_{\text {crit }}$, of the system performance $P(\mathcal{S}(\boldsymbol{q}))$ (e.g. a maximum tolerable displacement or stress in the structure), such as:

$$
P(\mathcal{S}(\boldsymbol{q})) \leqslant P_{\text {crit }} .
$$

- The robustness-to-uncertainty function, denoted $\hat{h}$, defining the greatest value of the uncertainty parameter $h$ for which the critical performance requirement is still ensured. This can be expressed by:

$$
\hat{h}=\max \left\{h \mid\left(\max _{\boldsymbol{q} \in \mathcal{U}\left(\mathbf{q}_{0}, h\right)} P(\mathcal{S}(\boldsymbol{q}))\right) \leqslant P_{\text {crit }}\right\} .
$$

In the following subsections, these components are explicitly derived within the scope of the design of prestressed solar panels, based on the finite element model described in Section 2.

\subsection{Performance requirement}

The performance requirement of interest in this study consists in ensuring that the panels remain in contact in order to avoid potentially damaging impacts. The associated condition on the local interface forces can be expressed as:

$$
\left\{\begin{array}{l}
\left|f_{\text {reac }}\right| \leqslant f_{p s} \\
f_{p s}>0
\end{array}\right.
$$

where $f_{p s}$ represents the considered static prestress load (assumed as positive while ensuring contact) that the reaction force of the snubber on the panel, $f_{\text {reac }}$, must not exceed. The three thresholds that will be studied are indicated in Fig. 3(b) where it is seen that the resonances at $89 \mathrm{~Hz}$ and $111 \mathrm{~Hz}$ may lead to the performance failure. This study will focus on the first resonance, with the highest amplitude. However, the extension to a frequency band containing several eigenmodes can be readily made.

It must be noticed that the non-linear dynamic response of the prestressed structure to corner impacts is not taken into account as the computations stop as soon as a loss of contact at the interface is reached. The physical system is thus reduced to a linear one.

All the reaction forces $f_{\text {reac }}$ that verify the previous performance criterion can be determined as a function of the frequency and the amplitude of the dynamic input force. This set, denoted $\mathcal{V}\left(f_{p s}\right)$,

$$
\mathcal{V}\left(f_{p s}\right)=\left\{\left(\omega, f_{\text {ext }}\right) \quad|\quad| f_{\text {reac }}\left(\omega, f_{\text {ext }}\right) \mid=f_{p s}\right\} .
$$

is bounded by curves that can be graphically obtained using Fig. 5(a), leading to Fig. 5(b). It can be observed that, for a given prestress load, the failure point corresponding to the lowest dynamic force $f_{\text {ext }}$, for which the panels separate, occurs at the resonance frequency. Indeed, with the increasing relative displacement of the corners, the reaction force increases and exceeds the prestress threshold value. Moreover, as expected, the higher the prestress load, the narrower $\mathcal{V}\left(f_{p s}\right)$ and thus the smaller the opportunity of failure.

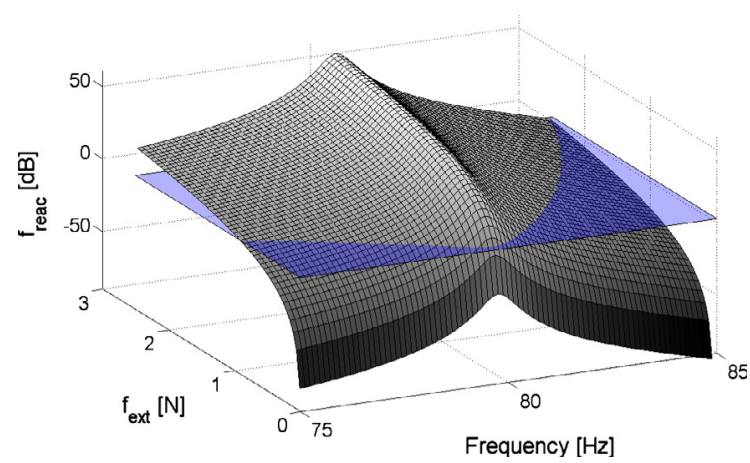

(a) Determination of the zone for $f_{p s}=2 \mathrm{~N}$

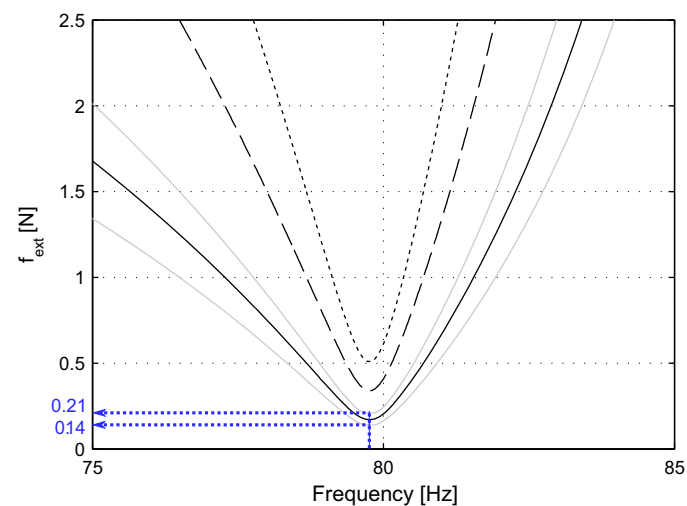

(b) Separation zone for $f_{p s}=(-) 2 N((-) \pm 20 \%)$, $(--) 4 N,(\cdots) 6 N$

Fig. 5. Separation zone $\mathcal{V}\left(f_{p s}\right)$ associated with the performance requirement. 


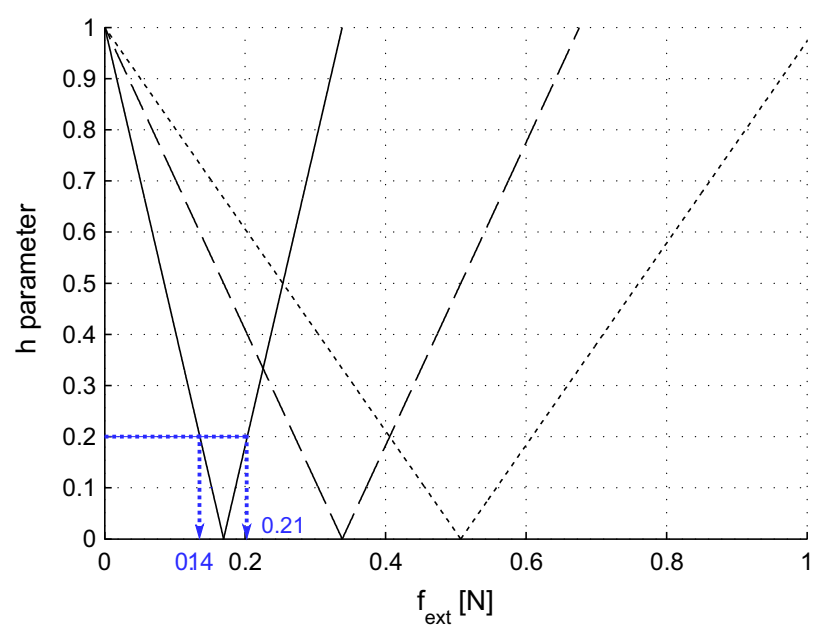

Fig. 6. Evolution of the failure point with uncertainty for $\tilde{f}_{p s}=(-) 2 N,(--) 4 N,(\cdots) 6 N$.

Finally, to handle the various sources and types of uncertainty detailed in the introduction and to comply with international standards, a common practice in the aerospace industry consists in adding safety factors at each step of the design process of a structure. Accordingly, the performance requirement criterion could be re-expressed as

$$
\left|f_{\text {reac }}\right| \leqslant s_{f} \cdot f_{p s}
$$

where $s_{f}$ is a safety factor, typically ranging from 1.05 to 1.2. It must be emphasized that the design procedure still remains deterministic while increasing the performance requirement: the larger the value of the safety factor, the safer the design. However, at this stage, it does not ensure any robustness of the targeted design to uncertainties. Unless otherwise indicated, this factor will remain equal to 1 .

\subsection{Uncertainty model}

As mentioned at the end of Section 1, the considered source of epistemic uncertainty is the prestress load value $f_{p s}$. Indeed, it is assumed here that the level of prestress induced by the snubbers is difficult to assess due to structural uncertainties (e.g. geometric defects, interface stiffness,...). Let $\tilde{f}_{p s}$ be the best estimate of $f_{p s}$ (assumed as positive). This quantity is a known but perhaps unreliable estimate of the corresponding value. Moreover, the extent to which it may be wrongly estimated is unknown. Such uncertainty can be modeled using a fractional error info-gap model, defined by

$$
\mathcal{U}\left(\tilde{f}_{p s}, h\right)=\left\{f_{p s} \quad \mid \frac{\left|f_{p s}-\tilde{f}_{p s}\right|}{\tilde{f}_{p s}} \leqslant h\right\}, h \geqslant 0 .
$$

The inequality states that the fractional error of the estimate is bounded by the horizon of uncertainty $h$ : when $h=0$, there is no uncertainty and the estimation is correct; as $h$ increases the uncertainty set becomes more and more inclusive. Since no realistic worst case is a priori known, $h$ is unbounded. The info-gap model is thus an unbounded family of nested sets representing possible realizations of $f_{p s}$.

Fig. 6 plots the evolution of the failure point in an uncertainty $v s$ dynamic force diagram. At $h=0$, the $f_{\text {ext }}$ value is the nominal one and the failure point corresponds to the inflexion point observed in Fig. 5(b). For each prestress load, two distinct curves originate from the initial estimate due to the absolute value in the uncertainty model, Eq. (6): the left branch pertains to the minimal value of $f_{e x t}$ and the right branch pertains to the maximal one. As an example, for $\tilde{f}_{p s}=2 N$ and $h=0.2$, the critical points can be obtained using the additional gray curves (labeled $\pm 20 \%$ ) Fig. 5(b) and following the blue ${ }^{2}$ dotted arrow lines.

From an engineering point of view the decreasing curves Fig. 6 correspond to the worst case of performance failure since the separation occurs for an input amplitude lower than the expected one. From a mathematical point of view, these curves represent the performance criterion given Eq. (5) where the unknown prestress load $f_{p s}$ has been replaced by its expression as a function of its best estimate and the horizon of uncertainty. Based on the chosen info-gap uncertainty model given Eq. (6),

$$
(1-h) \tilde{f}_{p s} \leqslant f_{p s} \leqslant(1+h) \tilde{f}_{p s}
$$

\footnotetext{
${ }^{2}$ For interpretation of color in Fig. 5, the reader is referred to the web version of this article.
} 
looking for the lower input amplitude amounts to considering the left-hand side equality. This finally implies decreasing levels of prestress load as the uncertainty increases. Therefore this study focuses on the left-hand parts of the curves.

These curves can also be a useful tool to investigate the tolerable level of uncertainty that sill avoids failure. For example, if the system is excited with a dynamic force $f_{\text {ext }}=0.17 \mathrm{~N}$, no uncertainty is tolerable if the prestress load is equal to $2 \mathrm{~N}$. Thus, the prestress force value must be exactly known. However, if $\tilde{f}_{p s}=4 \mathrm{~N}$, a 0.5 uncertainty level is now tolerable and even 0.68 in the last case, $\tilde{f}_{p s}=6 \mathrm{~N}$.

\subsection{Robustness definition}

The robustness of the system model considering uncertainty in the prestress load value can now be defined. This function, denoted $\hat{h}$, is the greatest horizon of uncertainty $h$ up to which the performance requirement is satisfied for all realizations of the uncertainty model:

$$
\hat{h}=\max \left\{h \mid\left(\max _{f_{p s} \in \mathcal{U}\left(\tilde{f}_{p s}, h\right)}\left(\left|f_{\text {reac }}\right|-s_{f} \cdot f_{p s}\right)\right) \leqslant 0\right\} .
$$

Considering the previous computations, representing this robustness function as a function of the reaction force would lead to the same plot as in Fig. 6, because of the linearity of the system model. Moreover, as previously highlighted, from an engineering point of view the worst case is reached for a minimal prestress value $\left(\tilde{f}_{p s}=2 N\right)$, zero uncertainty $(h=0)$ and a maximum value of the input force $\left(f_{\text {ext }}=0.17 \mathrm{~N}\right), c f$. Fig. 6 . The idea is therefore to study the influence of various safety factors on the robustness of this limit state.

The derived robustness curve is displayed in Fig. 7, in solid line. First, in agreement with the info-gap theory, it is confirmed that for $s_{f}<1$ the robustness remains null meaning that the performance requirement is not satisfied. However, from an engineering point of view, such values tend not to be considered as they would lead to underestimating the design constraint limit.

Secondly, higher safety factors imply higher degrees of robustness. The other two curves correspond to different estimated initial prestress values $\tilde{f}_{p s}$ but still pertain to the previous limit state. These confirm that, for a given safety factor, the higher the prestress value, the greater the robustness. Moreover, as observed for $\tilde{f}_{p s}=6 \mathrm{~N}$, the robustness curves tend to depict two different behaviors. For $s_{f}<0.8$, the slope is quite steep, indicating that a small increase of the safety factor implies an important gain in robustness. Conversely, for $s_{f}>0.8$, the slope becomes flat and there is no added value in further increasing the safety factor.

By restraining $s_{f}$ values to a typical industrial range (blue-shaded zone), it can be noted that the slope of the robustness curve is more important for $\tilde{f}_{p s}=2 \mathrm{~N}$. This confirms the fact that decreasing the safety factor as the design process progresses has more impact on the robustness when the structure is designed with tight performance margins (here for $\tilde{f}_{p s}=2 N$ ). More interestingly, by fixing the desired robustness level, e.g. to 0.3 , these curves show that: for $\tilde{f}_{p s}=2 \mathrm{~N}$, this amounts to consider a high safey factor $\left(s_{f}=1.43\right)$; for $\tilde{f}_{p s}=2 \mathrm{~N}$ or $6 \mathrm{~N}$, this robustness level is reached for all safety factor $s_{f}>1$. Hence, it is now

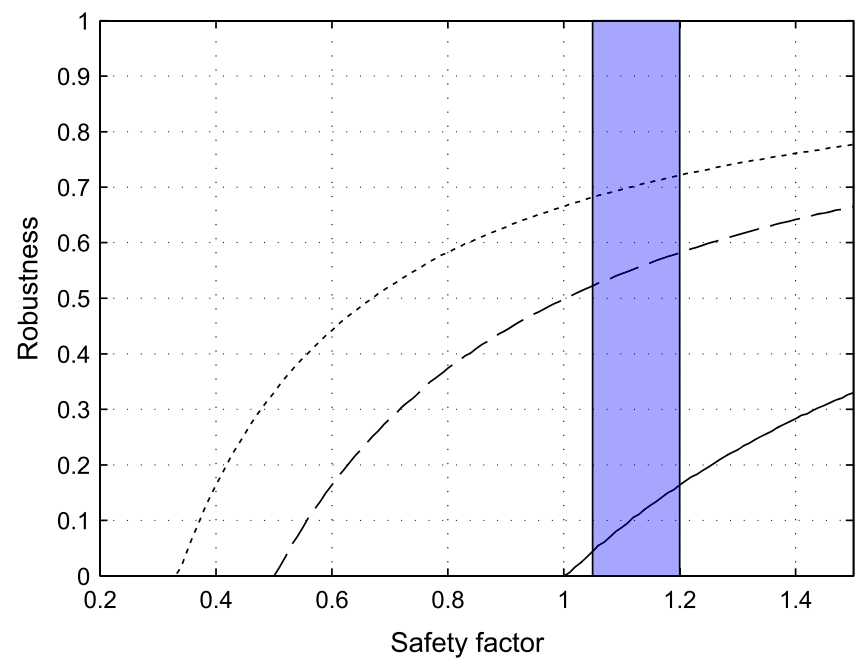

Fig. 7. Robustness of the performance requirement $v s$ safety factor for $\tilde{f}_{p s}=(-) 2 N,(--) 4 N,(\cdots) 6 N$. 
possible using such plots to derive the right value of the safety factor allowing to reach a chosen level of robustness: this may eventually lead to revise the aforementioned typical industrial range of safety factors.

\subsection{Comparison of different snubber stiffnesses}

In this last section, the stiffness of the snubbers is introduced as a third varying parameter. The associated reaction forces are computed for four different values of $k_{s}: 1 \times 10^{4}, 1 \times 10^{5}, 1 \times 10^{6}$ and $1 \times 10^{7} \mathrm{~N} \mathrm{~m}^{-1}$. As expected, softening or stiffening effects are observed, as shown in Fig. 8.

The same procedure as described below is successively applied to derive the robustness of the different designs. The obtained curves, displayed in Fig. 9, exhibit the same shape, which allows their respective robustness levels to be compared directly. Hence, it appears that, in this specific example, the stiffer case leads to the most robust design. However, a greater stiffness does not necessarily correspond to more robustness, as shown by the comparison between the $k_{s}=1 \times 10^{4} \mathrm{~N} \mathrm{~m}^{-1}$ and the $k_{s}=1 \times 10^{5} \mathrm{~N} \mathrm{~m}^{-1}$ configurations. This is mainly due to a modification of the global dynamic behavior of the structure with the snubber stiffness.

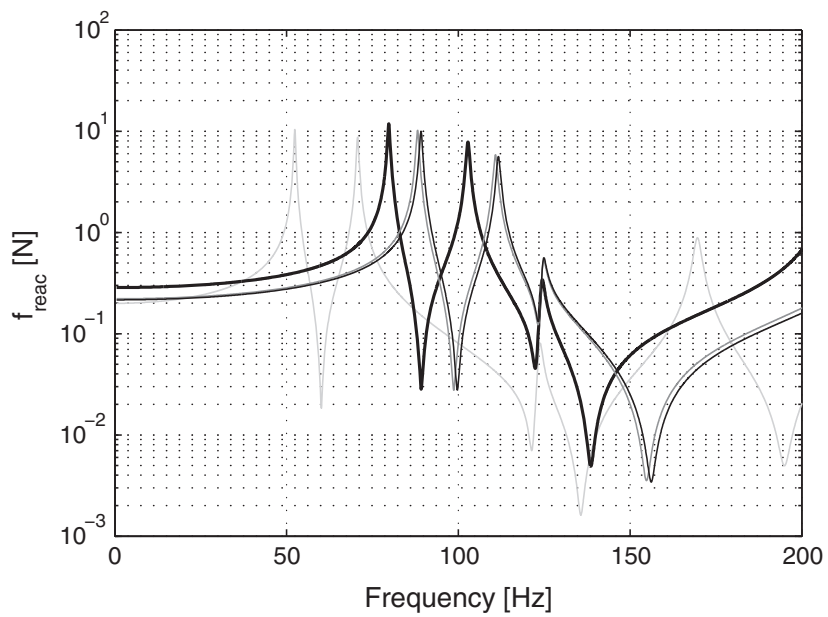

Fig. 8. Comparison of the reaction force at node 1 for different values of the snubber stiffness $k_{s}=(-) 1 \times 10^{4} \mathrm{~N} \mathrm{~m}^{-1},(-) 1 \times 10^{5} \mathrm{~N} \mathrm{~m}^{-1},(-) 1 \times 10^{6} \mathrm{~N} \mathrm{~m}^{-1}$ and $(-) 1 \times 10^{7} \mathrm{~N} \mathrm{~m}^{-1}$.

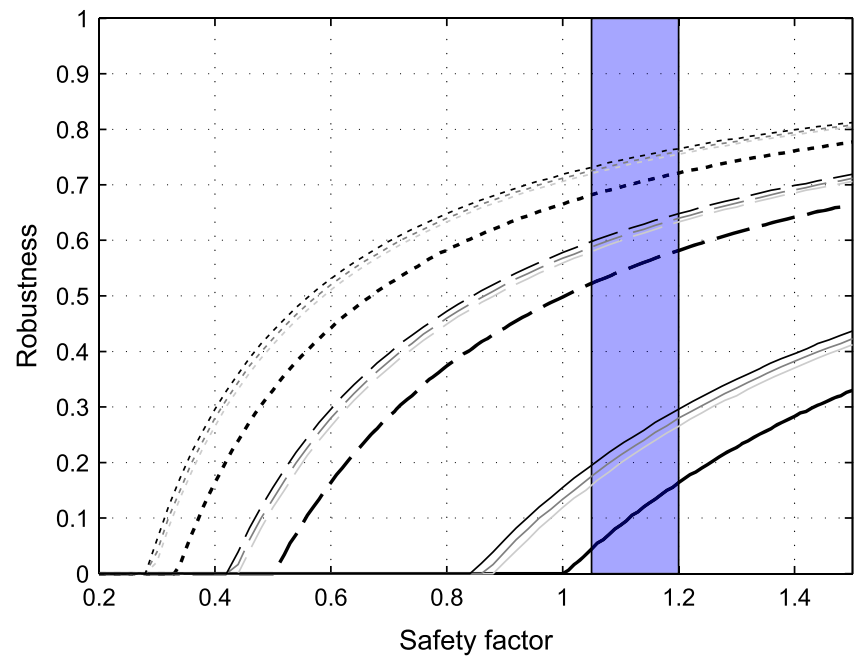

Fig. 9. Comparison of the robustness curves for different values of the snubber stiffness $k_{\mathrm{s}}=(-) 1 \times 10^{4} \mathrm{~N} \mathrm{~m}^{-1},(-) 1 \times 10^{5} \mathrm{~N} \mathrm{~m}^{-1}$, (-) $1 \times 10^{6} \mathrm{~N} \mathrm{~m}^{-1}$ and $(-) 1 \times 10^{7} \mathrm{~N} \mathrm{~m}^{-1}$; and $\tilde{f}_{p s}=(-) 2 \mathrm{~N},(--) 4 \mathrm{~N},(\cdots) 6 \mathrm{~N}$. 


\section{Conclusion}

A model-based indicator has been proposed in this paper to assess the robustness of the design of a prestressed structure representing solar arrays of satellites in their stowed configuration. Given the presence of severe uncertainty on the nominal prestress values, induced by the introduction of dedicated snubbers, an info-gap approach has been used to study the robustness of the critical design performance (i.e. non-separation of the panels) to lack of knowledge in the prestress. The influence of various parameters has been investigated and it has been shown that the proposed methods allow different design configurations to be compared and ranked, based on robustness considerations.

Another point addressed in this paper is the relationship between the safety factor and the level of robustness. Using the proposed methodology, it is now possible to relate the engineer's standard design margin parameter, or safety factor, to the concept of robustness. This also makes it possible to assess and/or to revise the considered values of these factors depending on the design process progress, the uncertainty level associated to the remaining design parameters and the targeted level of robustness.

Although straightforward, the application of this methodology to industrial structures would benefit from more detailed finite element models. By taking into account more realistic dynamic behaviors (e.g. prestressed, non-linear) the corresponding robustness curves might exhibit different shapes and several competing robust configurations could exist, depending on the assumed uncertainty level. Moreover, dedicated experiments could be performed to help define more accurately the uncertainty model, which remains the key component of the info-gap approach.

\section{Acknowledgment}

The work presented in this paper has been carried out with the generous support of the Centre National d'Etudes Spatials (Toulouse, France) and Thales Alenia Space (Cannes La Bocca, France). The authors would also like to thank professor Yakov Ben-Haim for his helpful remarks.

\section{References}

[1] R. Fey, B. Winter, J. Wijker, Sine sweep and steady-state response of a simplified solar array model with nonlinear support, in: DETC'99 ASME Design Engineering Technical Conferences, Las Vegas, Nevada, USA, pp. 1-12.

[2] H.-G. Beyer, B. Sendhoff, Robust optimization - a comprehensive survey, Comput. Meth. Appl. Mech. Eng. 196 (2007) $3190-3218$.

[3] G. Schuëller, H. Jensen, Computational methods in optimization considering uncertainties - an overview, Comput. Meth. Appl. Mech. Eng. 198 (2008) $2-13$.

[4] G. Schuëller, On the treatment of uncertainties in structural mechanics and analysis, Comput. Struct. 85 (2007) $235-243$.

[5] C. Soize, Random matrix theory for modeling uncertainties in computational mechanics, Comput. Meth. Appl. Mech. Eng. 194 (2005) 1333-1366.

[6] R. Moore, R. Kearfott, M. Cloud, Introduction to Interval Analysis, Society for Industrial and Applied Mathematics, Philadelphia, PA, USA, 2009.

[7] O. Dessombz, F. Thouverez, J.-P. Laîné, L. Jézéquel, Analysis of mechanical systems using interval computations applied to finite element methods, J. Sound Vib. 239 (2001) 949-968.

[8] L. Zadeh, Fuzzy sets, Inform. Control 8 (1965) 338-353.

[9] S. Rao, J.P. Sawyer, Fuzzy finite element approach for analysis of imprecisely defined systems, AIAA J. 33 (1995) $2364-2370$.

[10] P. Ladevèze, G. Puel, T. Romeuf, Lack of knowledge in structural model validation, Comput. Meth. Appl. Mech. Eng. 195 (2006) 4697-4710.

[11] Y. Ben-Haim, Info-gap value of information in model updating, Mech. Syst. Signal Process. 15 (2001) $457-474$.

[12] Y. Ben-Haim, Info-Gap Decision Theory: Decision Under Severe Uncertainty, second ed., Academic Press, Oxford, United Kingdom, 2006.

[13] Y. Ben-Haim, A. Laufer, Robust reliability of projects with activity-duration uncertainty, J. Constr. Eng. Manage. 124 (1998) $125-132$.

[14] F. Hemez, Y. Ben-Haim, Info-gap robustness for the correlation of test and simulations in non-linear transient, Mech. Syst. Signal Process. 18 (2004) $1443-1467$.

[15] S.C.P. Vinot, G. Lallement, Approche non-probabiliste de fiabilité basée sur les modèles convexes, Méc. Inud. 4 (2003) $45-50$.

[16] M. Guedri, S. Cogan, N. Bouhaddi, Robustness of structural reliability analyses to epistemic uncertainties, Mech. Syst. Signal Process. 28 (2012) 458469.

[17] S.C.P. Vinot, V. Cipolla, A robust model-based test planning procedure, J. Sound Vib. 288 (2005) 571-585.

[18] I. Takewaki, Y. Ben-Haim, Info-gap robust design with load and model uncertainties, J. Sound Vib. 288 (2005) 551-570. 